\title{
Ide-cabe: chili varieties identification and classification system based leaf
}

\author{
Wiwin Suwarningsih ${ }^{1}$, Purnomo Husnul Khotimah ${ }^{1}$, Andri Fachrur Rozie ${ }^{1}$, Andria Arisal ${ }^{1}$, Dianadewi \\ Riswantini $^{1}$, Ekasari Nugraheni ${ }^{1}$, Devi Munandar ${ }^{1}$, Rinda Kirana ${ }^{2}$ \\ ${ }^{1}$ Research Center for Informatics, National Research and Innovation Agency, Bandung, Indonesia \\ ${ }^{2}$ Indonesian Vegetable Research Institute (IVegRI), Bandung, Indonesia
}

\begin{tabular}{l} 
Article Info \\
\hline Article history: \\
Received Jul 8, 2021 \\
Revised Sep 8, 2021 \\
Accepted Jan 12, 2022 \\
\hline Keywords: \\
Chili variety \\
Deep CNN \\
Deep learning \\
Leaf identification \\
Variety classification
\end{tabular}

\begin{abstract}
Identifying good quality chili varieties can be done by observing their leaves. It is required for seed testing and certification processes. Currently, a manual leaf identification method is used in which human experts inspect a wide range of leaves every one to two months. An automatic method could increase the identification process. Deep learning has proven to be a prominent method for image classification. We investigate the performance of deep CNN models, as: AlexNet, VGG16, Inception-v3 and DenseNet-121; to classify chili variety. In this paper, we took images of leaves aged 10 days. A preprocessing strategy was taken to enrich the dataset and to boost the classification performance and to assess the proposed models' quality. From this study, we acquired 12 classes of chili leaves dataset. We acquired performance accuracy ranging from $70.18 \%$ to $78.37 \%$. Further, the classification results by DenseNet-121 obtained the highest accuracy of $78.37 \%$ and recall of $74.83 \%$. The classifiers investigated in this study perform well despite the relatively small number of our dataset. These results encourage the application of such an approach in practice.
\end{abstract}

This is an open access article under the CC BY-SA license.

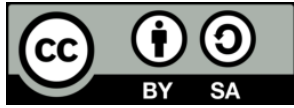

\section{Corresponding Author:}

Wiwin Suwarningsih

Research Center for Informatics, National Research and Innovation Agency

J1. Sangkuriang 21, Bandung 40135, Indonesia

Email: wiwi005@brin.go.id

\section{INTRODUCTION}

Red pepper (Capsicum annuиm L) is one of the most commonly cultivated vegetables due to its versatility and high economic value. Most of the uses of this vegetable commodity is for household needs, whether consumed in fresh, dry, or processed form. Also, red chilies are used as raw materials for the pharmaceutical industry. The data revealed by the Directorate General of Horticulture showed that in 2007 the planting area for red chilies in Indonesia reached 113,079 ha with a total production of 736,019 tones, with an average productivity of 6.51 tones/ha, viewed as still low when compared to potential yields ranging from 12-20 tons/ha. The increasing market demand for red chilies has encouraged the seed producers to provide quality plant seeds. The requirements for selecting plant varieties are based upon the market demand (taste, color, appearance, or size), high productivity, resistance to plant pests and sustainability for planting in local ecosystem conditions. The process of identifying different chili in current cultivars is still manual by looking at the leaves of each tree in the planting area. This manual method is dependent on human expertise. Hence, the method is prone to human error. In addition, the area coverage is quite vast. In West Java itself, one expert should cover two to three farms. However, the number of human experts is very scarce. Hence, an automatic strategy is required to support the process of sorting plants according to their varieties. 
Methods of cellular and molecular biology are used as automated methods to identify cultivars [1]. However, these methods are expensive and require very high energy from the environment, so they do not support enzymes for the molecular validation process compared to manual leaf identification methods [2], [3]. Using image processing techniques, leaf images can be easily processed and features can be directly extracted [4]. Describing the leaf shape requires a special approach or adaptation of the generic shape to a particular leaf box; apart from the shape of the leaves, this type of plant also has a unique leaf with a distinctive texture and leaf edges [5]. The leaf recognition process for classifying plant species is a problem that cannot be solved by algorithms alone, but requires a long learning process [6].

Investigations into the identification of images, for example, to distinguish the textures of images, have been carried out for a long time. Computers cannot distinguish textures that humans can see, so image textures can be distinguished by density, regularity, uniformity, and roughness [7], [8]. Thus, texture analysis is used to define patterns in digital images and generates a set of feature values or texture features that can later be computerized for the classification process [9].

Another approach, machine learning (ML) and computer vision techniques are widely used to determine the quality of agricultural products. Su et al. [8] evaluated the quality of potatoes after harvest according to their shape and size using a system we developed to predict the characteristics of potatoes based on machine vision. Wu and Zhou [10] conducted on the classification and ranking of fruit quality based on machine vision. Liming and Yanchao [7] conducted a study on a strawberry fruit grading system using the Kmeans clustering method in 2010. Various ML techniques used such as support vector machine (SVM), KNN, and artificial neural networks (ANNs) are evaluated on different types of fruits with different characteristics such as color, size, shape and texture. The maturity evaluation of mangosteen fruit was performed by combining RGB functions and implementing SVM to recognize maturity characteristics in the mangosteen fruit image [6]. The effectiveness of each ML method depends on the type of fruit being evaluated [11]. However, traditional ML technology still has several drawbacks, namely the error rate in the image recognition process as the feature extraction technology is done manually. One solution to this problem is to use deep learning techniques where feature extraction is performed automatically by an algorithm.

Deep learning technology that enables us to identify and classify leaf image patterns is an ANN [12]. ANN is one of the popular and reliable methods used to classify specific data with rather low computational load [13]. ANNs can study and analyze relationships between some sample data and recognize other similar data, but this has not been studied in artificial neural networks [14].

Classification using ANN is done with the following steps: mapping some new patterns as input data to produce output, storing the results of the pattern to enable them to be reused, performing optimization when finding similar patterns and making predictions of similar pattern mapping results [15], [16]. There are many types of ANN used for image data processing, i.e. general regression neural network (GRNN) is able to perform an optimization process to prevent any iterations by adjusting the data to only one smoothing parameter [17], [18]; backpropagation neural network (BNN) refers to an artificial neural network as a supervised learning method using a multi-layer network, and has the feature of minimizing the error in output generated in the network [19]-[21]; probabilistic neural network (PNN) refers to a classification method for a number of other categories [22], [23]; and convolutional neural network (CNN) is a neural network with an ability to classify image data [24], [25].

Deep CNN (DCNN) is a popular method to solve identification tasks in smart farming. The AlexNet method has been widely used in several studies to perform classification on image data such as [26], [27] to determine the quality of strawberries, the accuracy value obtained in this study is $96.48 \%$ in 2 classes, and $87.37 \%$ in 4 classes. Another classification study using AlexNet was also performed [28], which found defects in the tissue and achieved an accuracy value of 90\%. Then, a study [29] to classify tree species using AlexNet was performed, and the result of this study showed an accuracy value of $96.7 \%$. Based on the research that has been described previously, the AlexNet method is considered to have good performance in the classification process. In addition, AlexNet is also considered to have a very good ability in recognizing and identifying a feature in image data.

Inception-v3 which is the first architecture with fewer parameters and efficient computation [27]. One of the advantages of Inception-v3 is that the classifier only requires low computation cost. In this V3 inception, there is factorization to reduce parameters. Even though the computation cost is already low, Inception-v3 cannot be used on devices with low computation resources. Another model is DenseNet, the DenseNet architecture in [30] can distinguish information, both on the network and stored. In previous research, it has been proven that DenseNet also requires less computation to achieve high performance. DenseNet was able to classify melanoma skin cancer with an average accuracy value of 94\% [31].

Based on the description, we attempted to apply DCNN to the problem of chili leaves classification to create smart agriculture for automatic identification of chili plant varieties. The contributions of this study: i) the development of data sets for varieties of chili leaves. The dataset was developed in collaboration with 
the Indonesian Vegetable Research Institute. The dataset is unique because it is a new dataset with a large number of classes. The dataset is essential for further development related, but not limited, to chili variety preservation; ii) the evaluation of existing DCNN classifiers against this new dataset.

The remainder of this paper is organized as: the second section is the research method that describes the research workflow used in this study; the results were discussed and analyzed in the "results and discussion" section. Finally, we conclude the paper in the "conclusions" section.

\section{METHOD}

The research workflow in this study was carried out in five steps: i) dataset collection; ii) image acquisition; iii) preprocessing strategy; iv) augmentation and v) DCNN model classifiers.

\subsection{Dataset collection}

Dataset is collected when the chili is aged 10 days. This leaf age is chosen based on the expert recommendation that is the age when the expert examines the chili leaves. This is an image captured by filming a chili tree and chili leaf at a planting site. We acquired 495 images from the first collection and 619 images from the second collection. Figures 1(a) to (1) shows examples of images taken from the first collection for each chili variety.

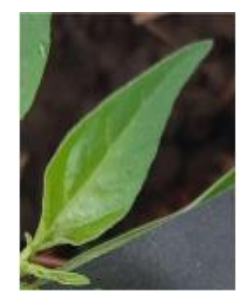

(a)

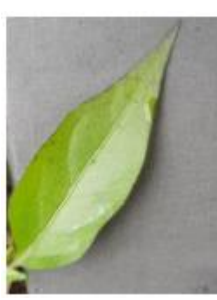

(g)

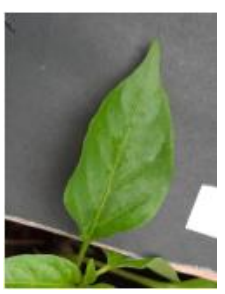

(b)

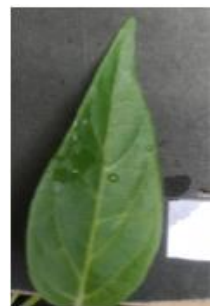

(h)

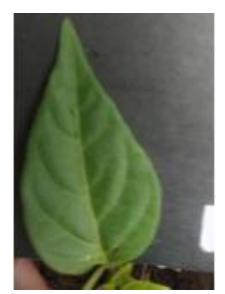

(c)

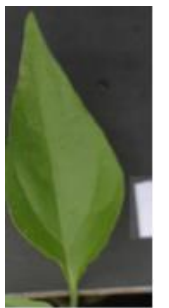

(i)

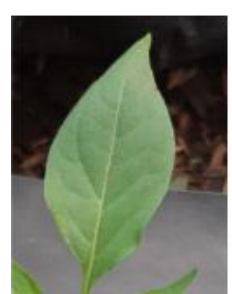

(d)

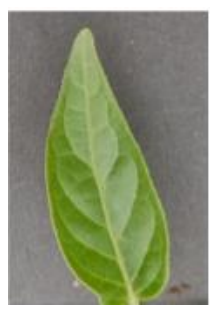

(j)

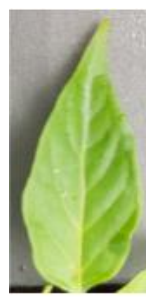

(e)

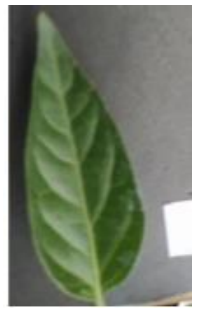

(k)

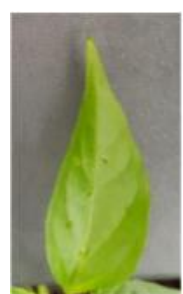

(f)

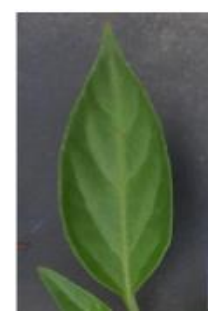

(1)

Figure 1. An example of a leaf dataset for 10-days dataset, (a) tanjung-1, (b) tanjung-2, (c) branang, (d) ciko, (e) lingga, (f) inata agrihorti, (g) carvi, (h) hot beauty, (i) carla agrihorti, (j) mia, (k) pilar, and (l) hot vision

\subsection{Image acquisition}

We used mobile phones and tablets with different resolutions for acquisition tools to enrich our dataset and obtain various kinds of leaf images with various resolutions. Each cell phone camera took a picture of every tree coming from a different variety. To prevent any damages in the condition of the plant, the data collection process was carried out with a minimum distance of $10 \mathrm{~cm}$. Also, images were taken randomly based on the captured images. Because the research object was outdoor, we then adjusted the lighting technique with an umbrella tool so that there was no leaf shadow that could affect the segmentation process and the identification of leaf object shapes. For the 10-day-old leaf image, we used two types of image backgrounds: a natural background and a black background. The preprocessing results showed that the natural background produced better images compared to the black background.

\subsection{Preprocessing strategy}

Pre-processing is the initial stage to prepare the raw data for the training process to enable quickly data classification [9], [15]. Our preprocessing stage aimed to remove any noise in the form of an unsuitable background. For this, the process included the cropped image data with a focus per leaf. By segmenting according to the shape and size of the leaves, we continued by clarifying the characteristics of the image data of the leaves. Reducing or enlarging the data size was based on the raw data obtained during observation. Subsequently, we converted the original data to obtain data based on the needs. Converting pixel color information into simple 
geometric objects was the vectorization of bitmap images [12]. Primitive graphical expression processes (such as lines, circles, and curves) are the common forms of variation from detecting the edges of an image by looking at similar brightness or color. The preprocessing procedure is shown in Figure 2.

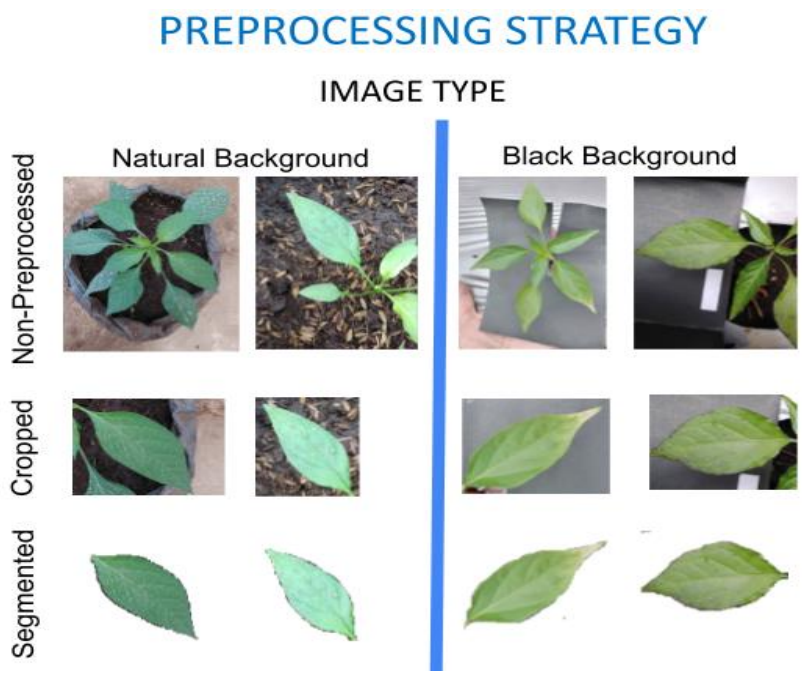

Figure 2. Preprocessing strategy

From the results of this segmentation, images with a white background are easy to identify by the system. RGB was considered an additive color space, and colors could be imagined as the result of an amount of bright red, blue, and green light onto a black background. The segmentation we did was done automatically using libraries from python. The libraries used included the cvtColor and cv2.COLOR_RGB2GRAY commands where cvtColor is a command to convert colors, cv2.COLOR_RGB2GRAY is a command to convert a color image to gray where the function is in the cv2 library.

\subsection{Augmentation}

One common characteristic of deep learning is the use of big data. Expansions are performed through multi-condition training to overcome a small collection [28], [29]. The procedure is by adding leaf images from various positions automatically, namely augmentation using the python library [30], [31]. We used methods such as $\mathrm{Wu}$ [23] to depict the digital morphology of the leaves. The first thing to do was to get the basic geometric properties of the sheet, such as: i) further define the distance between two points on the contour of the sheet represented by D; ii) defines the distance between the two terminals of the sheet image represented by L; iii) draw a vertical line on the two main ports. The maximum distance between intersections was defined as the width indicated by W. If the angle formed was $90^{\circ} \pm 0.5^{\circ}$, the two lines were considered orthogonal because the coordinates of the pixels were discrete. Relationship between length and width; iv) count the number of pixels with a value of 255 in the smoothed sheet image indicated by $A$; v) Calculate the number of pixels at the edge of the sheet image indicated by $\mathrm{P}$.

\subsection{DCNN model classifiers}

We used Keras as a deep learning library in building the DCNN model. Four classifier models are developed as:

a. AlexNet [32]

The AlexNet architecture consists of eight layers, the first five are convolution layers, with the largest pooling following the first, second, and fifth convolution layers. The output of this layer proceeds to three fully connected layers. AlexNet uses rectified linear units (ReLU) as an activation function which has the advantage of lesser training time compared to tanh. AlexNet also supports multi-GPU training by putting half of the model's neurons on one GPU and the other half on another GPU, resulting in faster training time. The overlapping max pooling in AlexNet has also proven to make the model harder to overfit.

b. VGG16 [33]

VGG16 architecture consists of sixteen convolutional layers with very small $3 \times 3$ convolutional filters. The first and second groups of two convolutional layers are maximally merged and the next 4 convolutional layers are maximally merged. Then there was a stack of folding layers followed by three 
fully connected layers. VGG16 uses ReLU as the common activation function within the hidden layers and a Soft-Max layer as the final layer.

c. Inception-v3 [34]

Inception-v 3 is the third iteration of the inception layer family. The inception architecture is a sparsely connected architecture where convolutional layers $(1 \times 1,3 \times 3$, and $5 \times 5)$ are concatenated into a single output vector. It introduces model regularization using label smoothing.

d. DenseNet-121 [35]

DenseNet121 consists of four high density pools, each high density pool followed by a 1x 1 convolution layer and an average pool layer. Each dense block consists of multiply $1 \times 1$ and $3 \times 3$ convolutional layers, as many as $6,12,32$, and 32, respectively. The output of dense blocks was forwarded as input of the next dense block. DenseNet-121 also uses ReLU as the common activation function within the hidden layers. Its classification layer consists of a global average pool and a Soft-max layer as the final layer.

\section{RESULTS AND DISCUSSION}

In this section, we present the results of the evaluation in this study. The results of the training and validation of the performance of the onsite model are shown in Figure 3 and Figure 4 . The purpose of this training is to select the best model so that it can improve the performance of the model against the datasets that we have. The observed values are accuracy and loss. Therefore, the DCNN model that achieves the highest accuracy value is considered the most suitable architecture for the classification of plant varieties. Empirically, Figures 3 and 4 require 50 epochs. The number of epochs is a hyperparameter that determines how many times the learning algorithm will work to process the entire training dataset and where the values of accuracy and loss in the training and validation processes converge. Epoch means that each sample in the training dataset has a chance to update the parameters of the internal model. Both figures show that overall, Inception-v3 and DenseNet-121 were outperformed compared to the others, delivering higher accuracies and lower losses. Moreover, Inception-v3 resulted in more stable performance than DenseNet-121 after epoch 5.

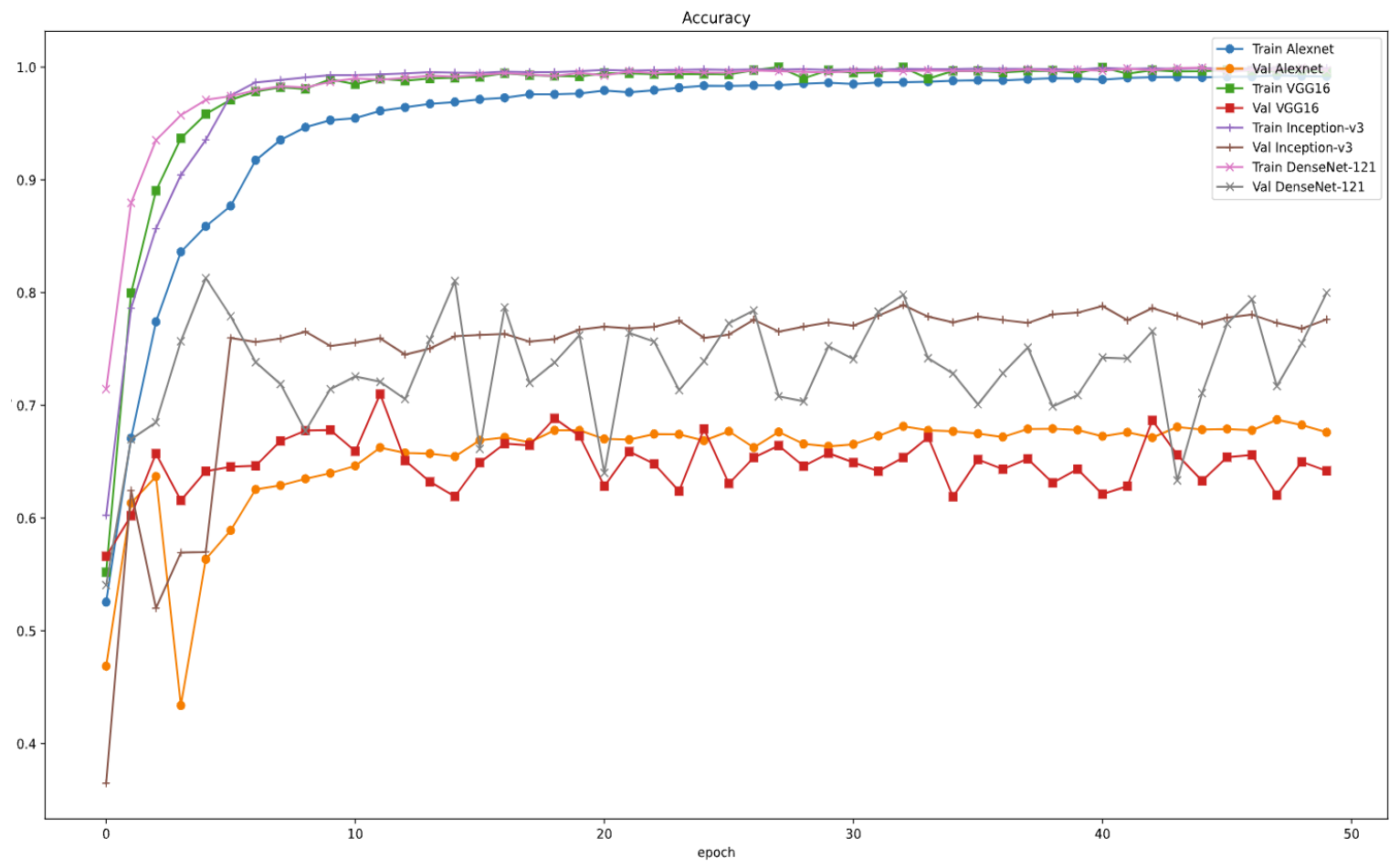

Figure 3. Training and validation accuracy of AlexNet, VGG16, Inception-v3 and DenseNet-121

In Figure 3, we could observe that DenseNet-121's training results outperformed the other three models, successively the best results were on Inception-v3, VGG16 and AlexNet. The accuracy of DenseNet121 is influenced by the depth of the layers, and DenseNet121 connects the input and output layers more tightly for efficient training. This is evidence that the advantages of DenseNet-121 can reduce the vanishing-gradient problem, strengthen feature propagation, and reduce the number of parameters as stated in [28]. 


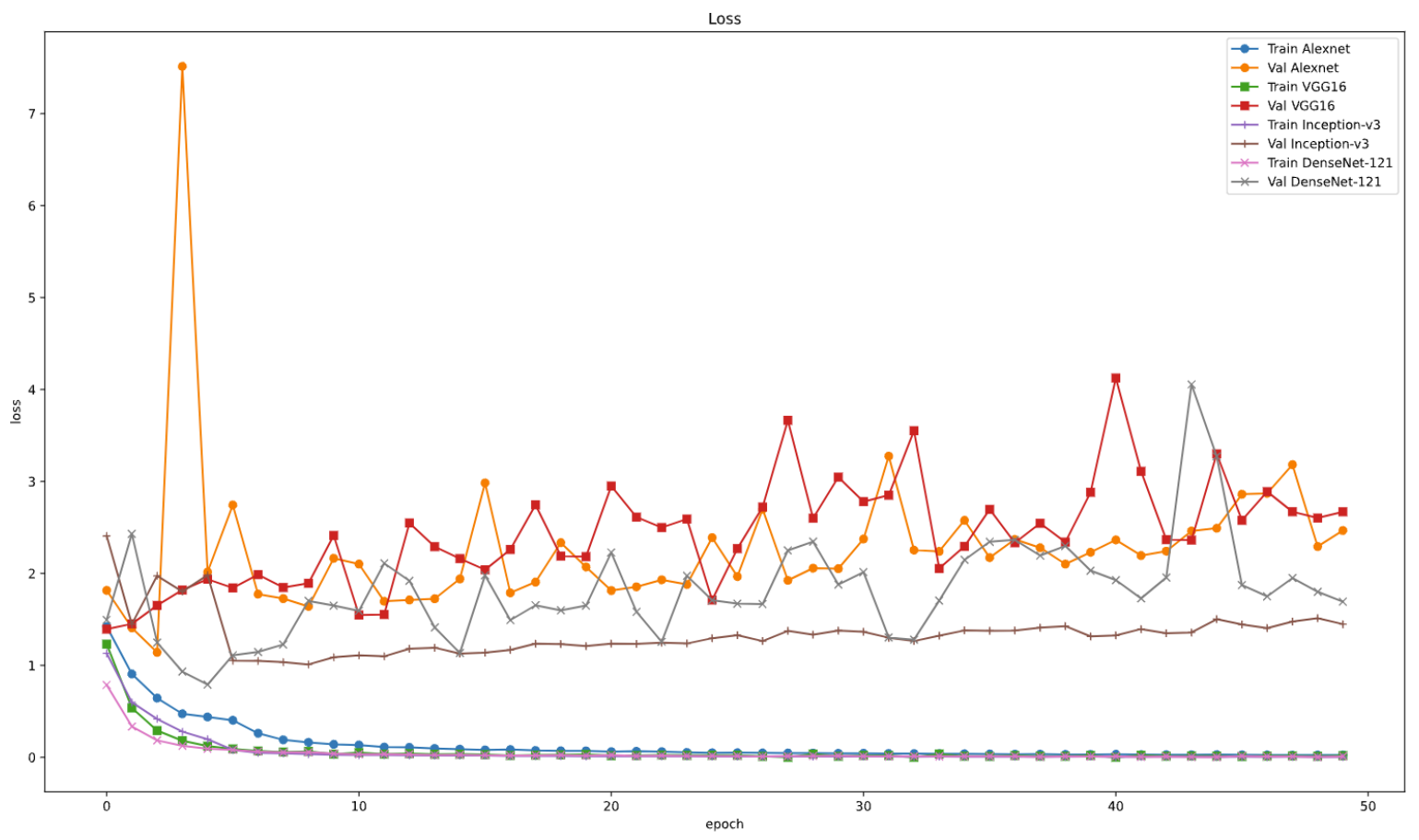

Figure 4. Training and validation loss of AlexNet, VGG16, Inception-v3 and DenseNet-121

Figure 4 shows that after 50 epochs, we get the loss (i.e., the value that the neural network is trying to minimize the distance difference between the actual label and the predicted label) for the training stage, the lowest value on the DenseNet-121 model while the highest on Alexnet. Meanwhile, in the validation stage, the lowest value is on the Inception-v3 model and the highest is on VGG16. Loss is a function that describes the loss associated with the probability generated by the DCNN model. The loss function used in this study is categorical cross-entropy. One of the categorical cross-entropy requirements that can be used is the classification process using the softmax activation function. Categorical cross-entropy is used when there is only one correct result. Category cross entropy compares the predicted probability distribution with the correct class (target) distribution, setting the correct class probability to 1 and setting the other classes to 0 .

The performance measurement of our leaf-based identification and classification system in this study used accuracy, precision, recall and F1-score metrics. The testing with a DenseNet-121 accuracy 78.37\%, and recall $74.83 \%$. Accuracy was used to measure the level of closeness between the predicted value and the actual one. The accuracy $78.37 \%$ value answered the question "What percentage of correct leaves are predicted based on the classification of varieties and not from the entire leaf dataset?". Meanwhile, recall was the success rate of the system in recovering information. The recall $74.83 \%$ value answered the question "What percentage of leaves were predicted according to the variety classification compared to the entire dataset of leaves that were actually classified".

Based on Table 1, the results of tests performed using accuracy, recall, and F1 scores were the lowest for VGG16, at $69.06 \%, 66.62 \%$, and $67.56 \%$, respectively. The precision and recall values indicate that the VGG16 model is poor in recognizing leaf varieties and the F1 value indicates that this model has large false negative and false positive values. As for the best value on precision, recall, F1 is in the DenseNet-121 model. These values can give an idea that the model build is quite good using the Densenet-121 model. If we look at the accuracy value, which shows the closeness of the measurement results to the real value, the highest value is found in the DenseNet-121 model, which is also $78.37 \%$. It shows that with a minimal dataset using a model of DCNN can produce better accuracy. DCNN's models such as DenseNet-121 and Inception-v3 are able to identify and classify chili leaf images better. In this study, we felt that there were still many shortcomings, especially for the limitations of our dataset. We were not able to collect image data until harvest due to COVID pandemic where there was a lockdown policy. Even so, the dataset we had was able to carry out the classification process for chili plant varieties. Our results are similar with [25] that DenseNet performances excel compared to other classifiers. 
Table 1. Testing result for 4 model CNN (AlexNet, VGG16, Inception-v3 and DenseNet-121)

\begin{tabular}{ccccc}
\hline Model & Accuracy $(\%)$ & Precision $(\%)$ & Recall $(\%)$ & F1 (\%) \\
\hline AlexNet & 70.18 & 72.80 & 67.36 & 68.95 \\
VGG16 & 70.70 & 69.06 & 66.62 & 67.56 \\
Inception-v3 & 75.68 & 74.59 & 72.60 & 72.57 \\
DenseNet-121 & 78.37 & 79.43 & 74.83 & 75.63 \\
\hline
\end{tabular}

\section{CONCLUSION}

This study presents a comparison of four models of DCNN, namely AlexNet, VGG16, Inception-v3 and DenseNet-121. Our research concludes that the best model is the DenseNet-121 with the highest accuracy of $78.37 \%$ and $\mathrm{F} 1$-score $75.63 \%$. But because a low dataset limits it with little variation, the values obtained are far from what we expected. The results of the research model with a neural network, we try to minimize errors. Thus, the objective function is often referred to as the loss function, which can also be used as an assessment of the performance of the observed model.

Interesting future directions for future research are given as: i) optimization of deep learning methods can be used to improve research on plant variety identification applications, such as identification through chili fruit or seeds used as plant seeds; ii) the classification performance of other data sets for other plant species can also adopt the methodology proposed in this study; iii) furthermore, to improve the accuracy of each analyzed model, it takes a long time to complete each epoch.

\section{ACKNOWLEDGEMENTS}

This research was conducted and funded by the Ministry of Research and Technology in the National Innovation System Research Incentive Program (INSINAS) with contract number: 12/INS/PPK/E4/2021 dated 26 April 2021. Data analysis related to deep learning was carried out by the informatics research center team the Indonesian Institute of Sciences, while the plant object was prepared by the Lembang Vegetable Research Institute.

\section{REFERENCES}

[1] V. Buchanan-Wollaston, "The molecular biology of leaf senescence," Journal of Experimental Botany, vol. 48, no. 2, pp. 181-199, 1997, doi: 10.1093/jxb/48.2.181.

[2] J. Nowosielski, W. Podyma, and D. Nowosielska, "Molecular Research on the Genetic Diversity of Polish Varieties and Landraces of Phaseolus Coccineus L. and Phaseolus Vulgaris L. Using the RAPD and AFLP Methods," Cellular \& Molecular Biology Letters, vol. 7, pp. 753-762, 2002.

[3] K. V. M. Rao, A. S. Raghavendra, and K. J. Reddy, Physiology and molecular biology of stress tolerance in plants, Switzerland: Springer, 2006.

[4] A. N. Rathod, B. Tanawal, and V. Shah, "Image Processing Techniques for Detection of Leaf Disease," International Journal of Advanced Research in Computer Science and Software Engineering, vol. 3, no. 11, pp. 397- 399, 2013.

[5] R. Hussin, M. R. Juhari, N. W. Kang, R. C. Ismail, and A. Kamarudin, "Digital image processing techniques for object detection from complex background image," Procedia Engineering, vol. 41, pp. 340-344, 2012, doi: 10.1016/j.proeng.2012.07.182.

[6] S. Riyadi, A. Zuhri, T. K. Hariadi, I. Prabasari, and N. A. Utama, "Optimized estimation of mangosteen maturity stage using svm and color features combination approach,” International Journal of Applied Engineering Research, vol. 12, no. 24, pp. 1503415038, 2017.

[7] X. Liming and Z. Yanchao, "Automated strawberry grading system based on image processing," Computers and Electronics in Agriculture, vol. 71, suppl. 1, pp. S32-S39, 2010, doi: 10.1016/j.compag.2009.09.013.

[8] Q. Su, N. Kondo, M. Li, H. Sun, and D. F. Al Riza, "Potato feature prediction based on machine vision and 3D model rebuilding," Computers and Electronics in Agriculture, vol. 137, pp. 41-51, 2017, doi: 10.1016/j.compag.2017.03.020.

[9] T. Rumpf, A. K. Mahlein, U. Steiner, E. C. Oerke, H. W. Dehne, and L. Plümer, "Early detection and classification of plant diseases with Support Vector Machines based on hyperspectral reflectance," Computers and Electronics in Agriculture, vol. 74, no. 1, pp. 91-99, 2010, doi: 10.1016/j.compag.2010.06.009.

[10] Q. Wu and C. Zhou, "Feature Extraction and Automatic Recognition of Plant Leaf Using Artificial Neural Network," Advances in Artificial Intelligence, pp. 5-12, 2006.

[11] S. Singh, M. Markou and J. Haddon, "Natural object classification using artificial neural networks," Proceedings of the IEEE-INNSENNS International Joint Conference on Neural Networks. IJCNN 2000. Neural Computing: New Challenges and Perspectives for the New Millennium, 2000, pp. 139-144 vol.3, doi: 10.1109/IJCNN.2000.861294.

[12] S. Hati and S. G, "Plant Recognition from Leaf Image through Artificial Neural Network," International Journal of Computer Applications, vol. 62, no. 17, pp. 15-18, 2013, doi: 10.5120/10172-4897.

[13] S. B. Jadhav, V. R. Udupi, and S. B. Patil, "Convolutional neural networks for leaf image-based plant disease classification," IAES International Journal of Artificial Intelligence (IJ-AI), vol. 8, no. 4, pp. 328-341, 2019, doi: 10.11591/ijai.v8.i4.pp328-341.

[14] F. N. Fauziah and F. Helmi, "Classification Using Feature Extraction of Leaf Based on Neural Network Algorithm with K Nearest Neighbor and Supervised Learning," International Journal of Simulation Systems, Science and Technology (IJSSST), pp. 0-7, January 2019, doi: 10.5013/ijssst.a.20.06.14.

[15] C. L. Lee and S. Y. Chen, "Classification of leaf images," International Journal of Imaging Systems and Technology, vol. 16, no. 1, pp. 15-23, 2006, doi: 10.1002/ima.20063.

[16] S. B. Park, J. W. Lee, and S. K. Kim, "Content-based image classification using a neural network," Pattern Recognition Letters, vol. 25, no. 3, pp. 287-300, 2004, doi: 10.1016/j.patrec.2003.10.015. 
[17] Y. L. Murphey and Yun Luo, "Feature extraction for a multiple pattern classification neural network system," 2002 International Conference on Pattern Recognition, 2002, pp. 220-223 vol.2, doi: 10.1109/ICPR.2002.1048278.

[18] Teo Lian Seng, M. Khalid and R. Yusof, "Adaptive GRNN for the modelling of dynamic plants," Proceedings of the IEEE Internatinal Symposium on Intelligent Control, 2002, pp. 217-222, doi: 10.1109/ISIC.2002.1157765.

[19] Z. Zulkifli, P. Saad and I. A. Mohtar, "Plant leaf identification using moment invariants \& General Regression Neural Network," 2011 11th International Conference on Hybrid Intelligent Systems (HIS), 2011, pp. 430-435, doi: 10.1109/HIS.2011.6122144.

[20] J. W. Orillo, J. Dela Cruz, L. Agapito, P. J. Satimbre and I. Valenzuela, "Identification of diseases in rice plant (oryza sativa) using back propagation Artificial Neural Network," 2014 International Conference on Humanoid, Nanotechnology, Information Technology, Communication and Control, Environment and Management (HNICEM), 2014, pp. 1-6, doi: 10.1109/HNICEM.2014.7016248.

[21] M. Chanda and M. Biswas, "Plant disease identification and classification using Back-Propagation Neural Network with Particle Swarm Optimization," 2019 3rd International Conference on Trends in Electronics and Informatics (ICOEI), 2019, pp. 1029-1036, doi: 10.1109/ICOEI.2019.8862552.

[22] K. Dimililer and E. Kiani, "Application of back propagation neural networks on maize plant detection,” Procedia Comput. Sci., vol. 120, pp. 376-381, 2017, doi: 10.1016/j.procs.2017.11.253.

[23] S. G. Wu, F. S. Bao, E. Y. Xu, Y. Wang, Y. Chang and Q. Xiang, "A Leaf Recognition Algorithm for Plant Classification Using Probabilistic Neural Network,” 2007 IEEE International Symposium on Signal Processing and Information Technology, 2007, pp. 11-16, doi: 10.1109/ISSPIT.2007.4458016

[24] U. Krishnasamy and D. Nanjundappan, "Hybrid weighted probabilistic neural network and biogeography based optimization for dynamic economic dispatch of integrated multiple-fuel and wind power plants," International Journal of Electrical Power \& Energy Systems, vol. 77, pp. 385-394, 2016, doi: 10.1016/j.ijepes.2015.11.022.

[25] E. C. Too, L. Yujian, S. Njuki, and L. Yingchun, “A comparative study of fine-tuning deep learning models for plant disease identification," Computers and Electronics in Agriculture, vol. 161, pp. 272-279, 2019, doi: 10.1016/j.compag.2018.03.032.

[26] L. Perez and J. Wang, "The Effectiveness of Data Augmentation in Image Classification using Deep Learning," 2017, [Online]. Available: http://arxiv.org/abs/1712.04621

[27] S. Samir, E. Emary, K. El-Sayed, and H. Onsi, "Optimization of a pre-trained AlexNet model for detecting and localizing image forgeries," Information, vol. 11, no. 5, 2020, doi: 10.3390/INFO11050275.

[28] R. Sustika, A. Subekti, H. F. Pardede, E. Suryawati, O. Mahendra, and S. Yuwana, "Evaluation of deep convolutional neural network architectures for strawberry quality inspection," International Journal of Engineering \& Technology, vol. 7, no. 4, pp. 75-80, 2018, doi: 10.1007/s00216-016-9944-7

[29] K. K. Sudha and P. Sujatha, "A qualitative analysis of googlenet and alexnet for fabric defect detection," International Journal of Recent Technology and Engineering (IJRTE), vol. 8, no. 1, pp. 86-92, 2019.

[30] S. E. Spasov and P. Lio, "Dynamic Neural Network Channel Execution for Efficient Training," 2019, [Online]. Available: http://arxiv.org/abs/1905.06435.

[31] R. Refianti, A. B. Mutiara, and R. P. Priyandini, "Classification of melanoma skin cancer using convolutional neural network," International Journal of Advanced Computer Science and Applications (IJACSA), vol. 10, no. 3, pp. 409-417, 2019, doi: 10.14569/IJACSA.2019.0100353.

[32] Y. Zhang, J. Gao, and H. Zhou, "Breeds Classification with Deep Convolutional Neural Network," ICMLC 2020: Proceedings of the 2020 12th International Conference on Machine Learning and Computing, pp. 145-151, 2020, doi: 10.1145/3383972.3383975.

[33] I. Hachchane, A. Badri, A. Sahel, and Y. Ruichek, "Large-scale image-to-video face retrieval with convolutional neural network features," IAES International Journal of Artificial Intelligence (IJ-AI), vol. 9, no. 1, pp. 40-45, 2020, doi: 10.11591/ijai.v9.i1.pp4045 .

[34] C. Szegedy, V. Vanhoucke, S. Ioffe, J. Shlens and Z. Wojna, "Rethinking the Inception Architecture for Computer Vision," 2016 IEEE Conference on Computer Vision and Pattern Recognition (CVPR), 2016, pp. 2818-2826, doi: 10.1109/CVPR.2016.308

[35] G. Huang, Z. Liu, L. Van Der Maaten and K. Q. Weinberger, "Densely Connected Convolutional Networks," 2017 IEEE Conference on Computer Vision and Pattern Recognition (CVPR), 2017, pp. 2261-2269, doi: 10.1109/CVPR.2017.243.

\section{BIOGRAPHIES OF AUTHORS}

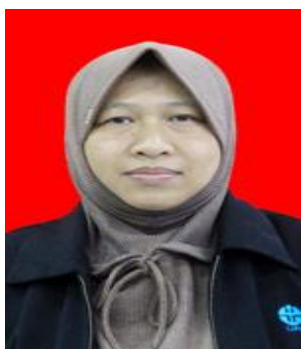

Wiwin Suwarningsih (D) SC P obtained her doctor degree from the Informatics department, Bandung Institute of Technology. Currently she is working as a researcher at National Research and Innovation Agency. Her research interests on Artificial Intelligence, natural language processing, text mining and question answering systems. She can be contacted at email: wiwi005@brin.go.id.

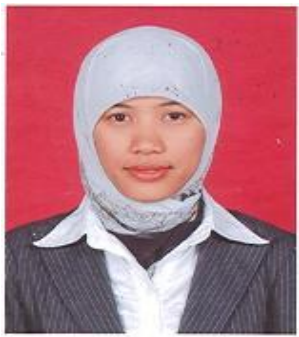

Purnomo Husnul Khotimah (iD) $8 \mathrm{SC}$ P obtained her P. Hd. degree from Kyoto University. Currently she is working as a researcher at National Research and Innovation Agency. Her research interests on data mining, information retrieval, data integration, information system, distributed information system, xml, webbased implementation, open source. She can be contacted at email: purn005@brin.go.id. 


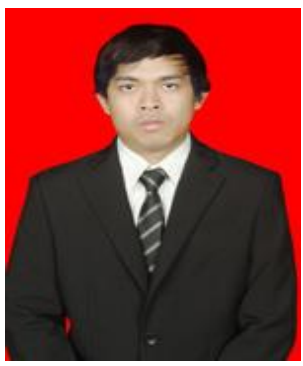

Andri Fachrur Rozie (D) SC P obtained his Master degree from Korea University. Currently he is working as a researcher at National Research and Innovation Agency. His research on text mining, software engineering, parallel computing, big data, and data mining. He can be contacted at email: andr035@brin.go.id.

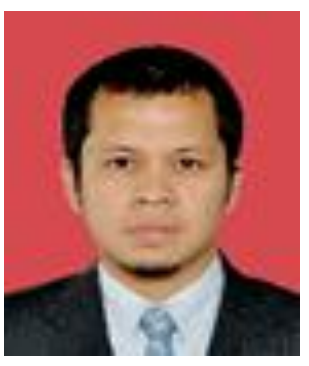

Andria Arisal (D) 8. SC P obtained his Master degree from University of Melbourne. Currently he is working as a researcher at National Research and Innovation Agency. His research on parallel computing, big data, and data mining. He can be contacted at email: andria.arisal@brin.go.id.

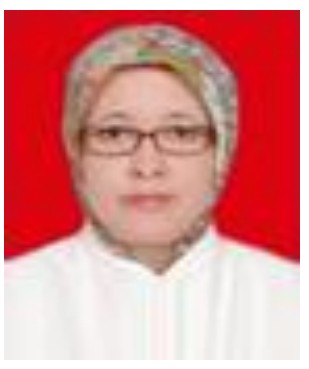

Dianadewi Riswantini (D) SC S P obtained her Master degree from Delft University of Technology. Currently she is still studying at School of Business and Management, Bandung Institute of Technology as a doctoral student. Currently she is working as a researcher at National Research and Innovation Agency. Her research interests are on data analytics, text mining, and NLP. She can be contacted at email: dian003@brin.go.id.

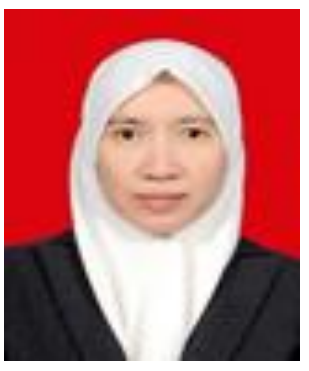

Ekasari Nugraheni (D) SC P obtained her Master degree from the Informatics department, Bandung Institute of Technology. Currently she is working as a researcher at National Research and Innovation Agency. Her research interest on data mining, data analytics, information retrieval, web programming and NLP. She can be contacted at email: ekas003@brin.go.id.

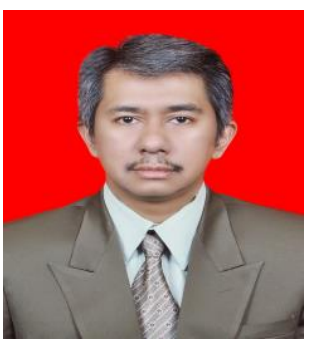

Devi Munandar (iD 8 SC P currently studying at Pajajaran University as a master student and he is working as a researcher at National Research and Innovation Agency. Her research interests are on text mining, software engineering and deep learning modelling. He can be contacted at email: devi002@brin.go.id.

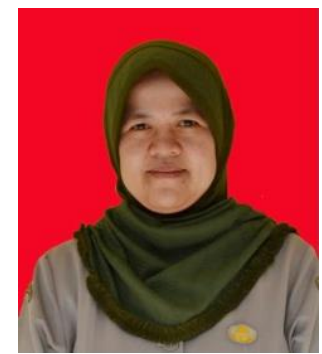

Rinda Kirana (iD 80 SC $\mathrm{P}$ obtained her doctor degree from Bandung Institute of Technology. Currently she is working as a researcher at Indonesian Vegetable Research Institute (IVegRI). Her research interests are on plant breeding and genetics in chili plants. She can be contacted at email: rindakirana@gmail.com. 